\title{
Communications and Networking for Smart Grid: Technology and Practice
}

\author{
Chi Zhou, ${ }^{1}$ Hossam S. Hassanein, ${ }^{2}$ Robert Qiu, ${ }^{3}$ and Pierangela Samarati ${ }^{4}$ \\ ${ }^{1}$ Department of Electrical and Computer Engineering, Llinois Institute of Technology, 3301 S. Dearborn Street, \\ Chicago, IL 60616, USA \\ ${ }^{2}$ School of Computing, Queens's University, Kingston, ON, Canada K7L 3N6 \\ ${ }^{3}$ Department of Electrical and Computer Engineering, Tennessee Tech University, Campus Box 5077, Cookeville, \\ TN 38505, USA \\ ${ }^{4}$ Department of Information Technology, University of Milan, Via Bramante 65, 26013 Crema, Italy
}

Correspondence should be addressed to Chi Zhou, zhou@iit.edu

Received 18 December 2011; Accepted 18 December 2011

Copyright () 2011 Chi Zhou et al. This is an open access article distributed under the Creative Commons Attribution License, which permits unrestricted use, distribution, and reproduction in any medium, provided the original work is properly cited.

The drive for improved power system efficiency, stability, and flexibility has served as a catalyst for smart grid research and development, so the communications networks in smart grid must facilitate the wide-scale utilization of various communications systems, enable the interoperability among various communications protocols, and provide secure and reliable communications for the smart grid. Many open issues in communications and networking need to be addressed by researchers.

The major interesting topics investigated in this special issue include home area networking (ZigBee, OpenHAN, etc.), wireless mesh networking, and neighborhood area networks, protocol interoperability, green routing and switching protocols, and cyber and physical security and privacy. Many thanks to the authors for submitting high-quality papers as well as to guest editors and reviewers for providing the timely reviews. A summary of papers is as follows.

"M2M communications in the smart grid: applications, standards, enabling technologies, and research challenges," by S. K. Tan et al., presents some of the ongoing standardization work in machine-to-machine (M2M) communications followed by the application of M2M communications to smart grid. The paper analyzes and discusses the enabling technologies in M2M and provides an overview of the communications challenges and research opportunities with a focus on wireless sensor networks and their applications in a smart grid environment.
"Location discovery based on fuzzy geometry in passive sensor networks," by R. W. et al., provides a solution to location discovery with uncertainty for passive sensor networks in the nation power grid. The approach of fuzzy geometry is introduced to investigate the fuzzy measurability. The interplay between fuzzy geometry of target localization and the fuzzy estimation bias for the case of fuzzy linear observer trajectory is analyzed in detail in sensor networks.

"Building automation networks for smart grids," by P. Yi et al., presents a framework for end-to-end interoperability in home and building area networks within smart grids. 6LoWPAN and the compact application protocol are utilized for network and application layer interoperability, respectively. A differential service medium access control scheme enables end-to-end connectivity. Several issues are also addressed, including interference mitigation, load scheduling, and security and possible solutions.

"Expected transmission energy route metric for wireless mesh senor networks," by Y. L. Jin et al., considers wireless mesh sensor networks for smart grid application, as mesh topology achieves high throughput and stable intercommunication. A new routing metric is designed to improve the energy balance of the whole network and extends the lifetime of wireless mesh sensor networks.

"Cyber security for smart grid, cryptography, and privacy," by S. Iyer, reviews different types of attacks to smart grid. The specific focus is on cyber security, as the smart grid uses 
high level of computation. It is shown that cryptography and key management techniques can be used to overcome these attacks. The paper also discusses the privacy of consumers as another important security concern.

"BVS: a lightweight forward and backward secure scheme for PMU communications in smart grid," by W. Ren et al., proposes a family of security schemes that are lightweight in terms of computation and storage for phasor measurement units (PMUs), including billed value-based scheme (BVS). Security analysis justifies that the proposed schemes, especially BVS, can attain the security goals with low computation and storage cost.

"Cognitive radio for smart grid: theory, algorithms, and security," by R. Ranganathan et al., introduces a novel concept of incorporating a cognitive radio network as the communications infrastructure for the smart grid. From the power system point of view, a supervised learning method is used for the automated classification of power system disturbances. The impending problem of securing the smart grid is also addressed, in addition to the possibility of applying FPGA-based fuzzy logic intrusion detection for the smart grid.

Chi Zhou

Hossam S. Hassanein

Robert Qiu

Pierangela Samarati 

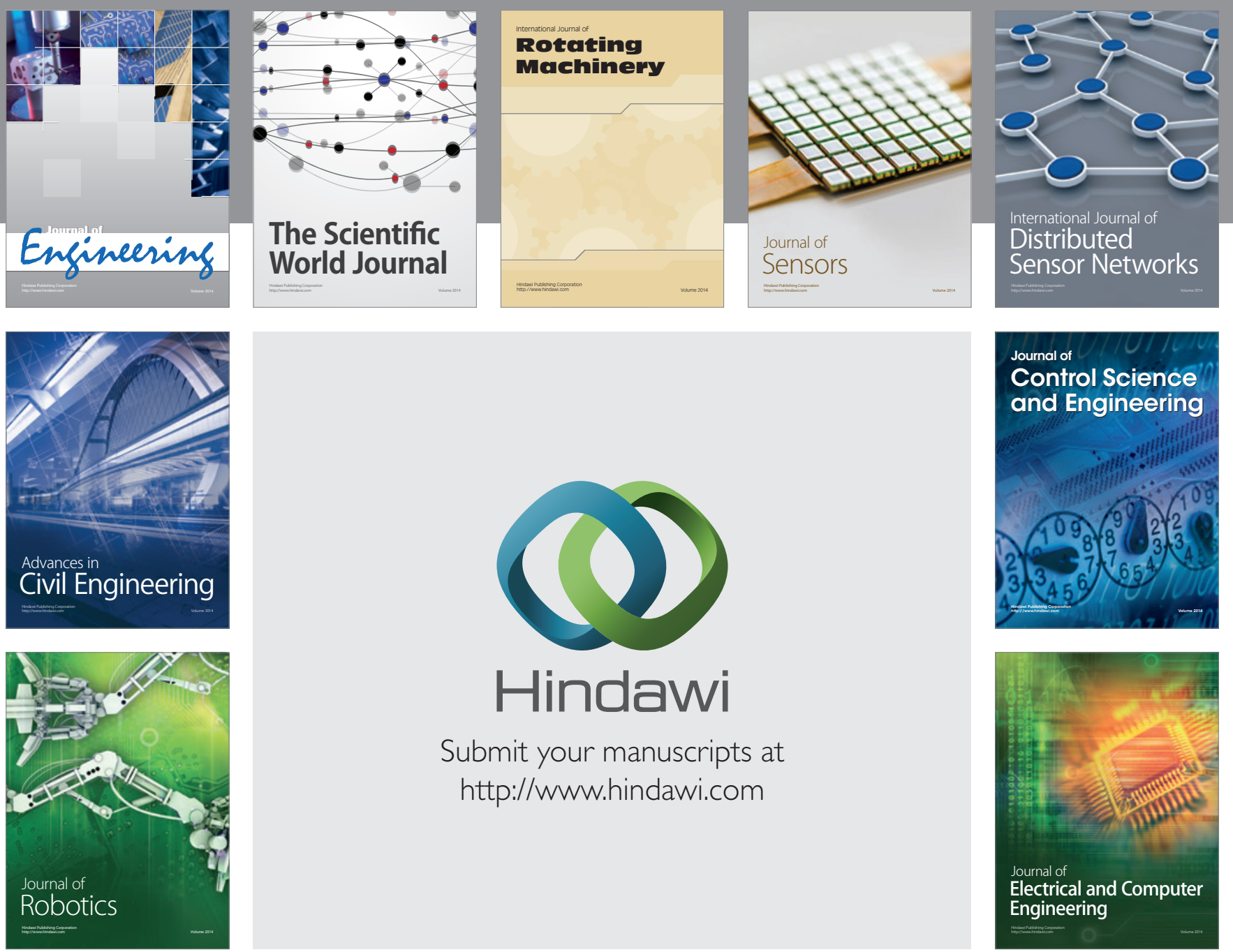

Submit your manuscripts at

http://www.hindawi.com
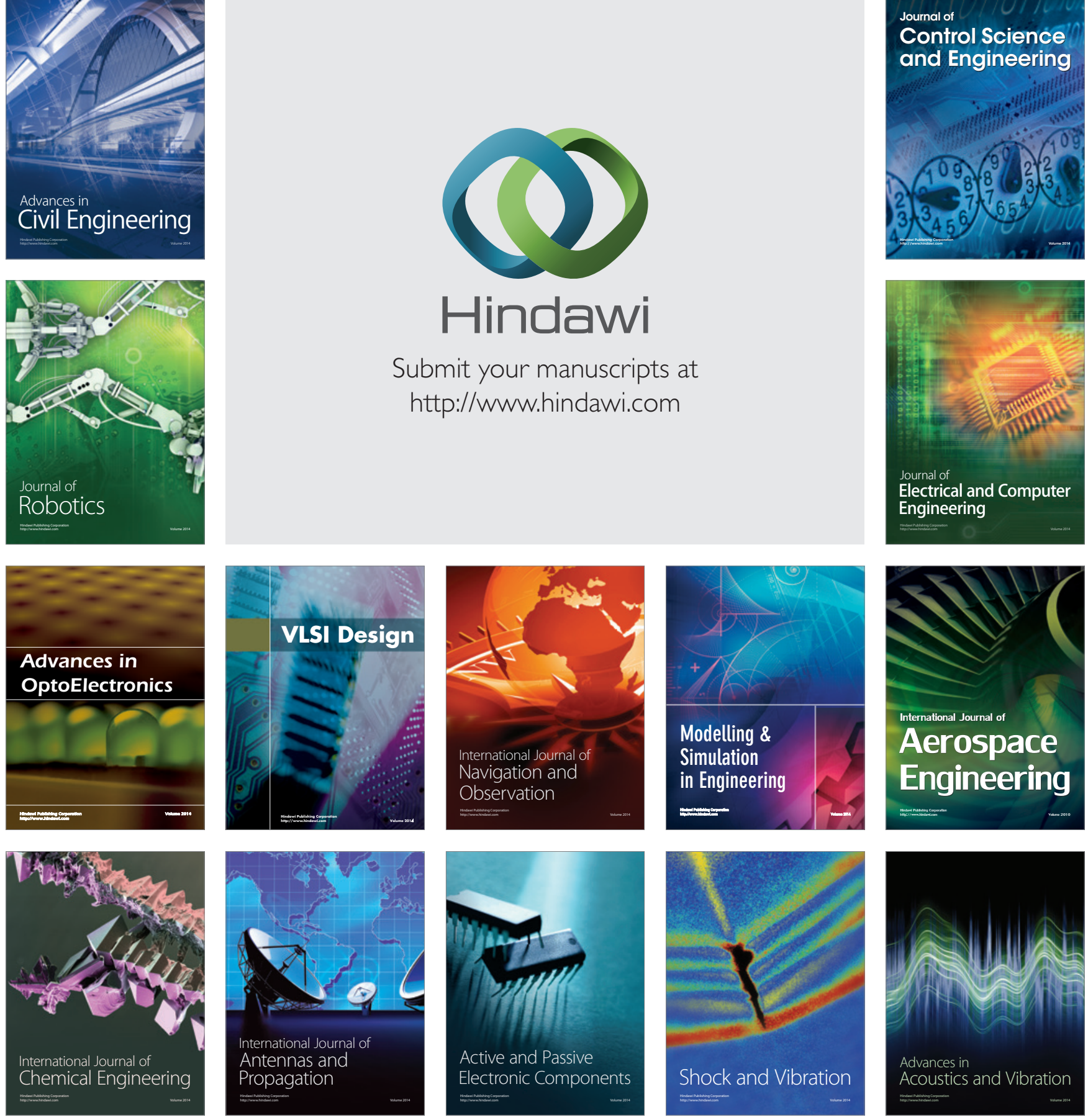